\title{
How Do We Have Feelings?
}

\author{
Irving C. Statler \\ Human Factors Research Division, NASA Ames Research Center, Moffett Field, CA, USA \\ Email: icstatler@earthlink.net
}

Received 24 August 2015; accepted 6 October 2015; published 9 October 2015

Copyright (C) 2015 by author and Scientific Research Publishing Inc.

This work is licensed under the Creative Commons Attribution International License (CC BY).

http://creativecommons.org/licenses/by/4.0/

c) (i) Open Access

\begin{abstract}
For centuries, the question of how a physical structure (the brain) generates the subjective feeling of consciousness has plagued neuroscientists, physiologists, psychologists, linguists, and philosophers. This has become known as the "hard problem of consciousness" and has been the subject of many publications. Although lots of answers have been proposed, none has been completely satisfactory. The focus of most of these studies has been on the neuronal structures and activities. Experiential consciousness emerges from neural processes, but it has not been explained with models that have been based solely on the electro-mechanical aspects of the processes. There must be some other dynamic features of neuronal activity to explain the emergence of experiential consciousness. I argue that a likely answer to the dilemma resides in the added dimension of the neurochemistry of the brain that has, so far, received little attention.
\end{abstract}

\section{Keywords}

Experiential Consciousness, Feelings, Mind-Body Problem, Hard Problem of Consciousness, Neuropharmacology

\section{Introduction}

How is it possible for a physical structure (your brain) to generate your subjective feeling of consciousness? How can anything material be conscious? How does a brain make a mind?

These questions have plagued neuroscientists, physiologists, psychologists, linguists, and philosophers for centuries [1] [2]. They constitute what David Chalmers [3] has called the "hard problem of consciousness".

The on-going search for the neuronal substrate of the subjective experience of consciousness has generated all sorts of speculative answers to these questions. You may consider, for example, Edelman's [4] [5] concept of "reentrant loops” or Humphrey’s [6] idea of "recurrent feedback loops” or Baars' [7] global workspace theory of consciousness or Penrose's [8] [9] sub-neuronal quantum-mechanical effects by the way of microtubules or Beck and Eccles' [10] [11] model based on quantum mechanics or Stapp’s [12] quantum theory. Crick and Koch 
[13] suggest that the sensations may arise from certain oscillations in the cerebral cortex by which processes in different parts of the brain become tied together by synchronized neural firings at 40 times per second. Many authors have proposed the concept of “qualia”, but the nature and existence of qualia remain controversial [14]-[17].

It is highly likely that many of these speculative proposals are, indeed, valid models of some of the non-conscious processes underlying conscious awareness. However, none of the proposals offered so far has provided a satisfactory solution to the riddle of the "hard problem of consciousness" and the so-called explanatory gap remains.

A contributing factor to the inadequacy of most of the current proposed solutions has been that they generally consider the term "experiential consciousness" to mean the feelings of cognition, attention, memory, emotion, or intention. But, as we seek an understanding of feelings, it is a mistake to limit ourselves to these. We also experience feelings of knowing that we are hungry, in pain, or nauseous or happy, or sad, or angry, or fearful, or disgusted. Dialogue, conversation, and communication stimulate feelings. Colors, odors, tastes, sounds, and touches can make us feel happy or sad [18] [19].

The question of how we have feelings pertains equally to any and all feelings that emerge from the brain regardless of which of our sensors signaled a change in our external environment or our internal bodily functions or our thoughts. I believe that our feeling that we know of our intention or our fear or our love emanates from the same aspects of neuronal processes, as do our feelings of hunger or thirst or pain or the redness of color or the loudness of sound or the stench of an odor [20]. The answer to the question we have posed must apply to all feelings from those we get with the smell of freshly baked bread to those associated with the long-awaited visit of a dear friend.

Experiential consciousness-feeling-emerges from neural processes, but the explanations based solely on the electro-mechanical aspects of the neuronal structures and operations offered so far have evidently not been satisfactory because new concepts continue to be proposed. Even though the recent studies may have developed valid models of non-conscious neural processes that underlie conscious experience, there must be some dynamic feature of neuronal activity in addition to the ones already addressed to explain how experiential consciousness can emerge.

\section{What Might Be Missing?}

I speculate that the feature of the neuronal processes that has not received sufficient attention is the neurochemistry. I propose that the subjective "feeling" of consciousness is a change in the state of the chemical balance in the brain. The "how" of a conscious experience is in the change in the distribution profile of the quantities, types, and locations across the brain of neurochemicals.

Evidence in support of my proposal comes from considerations of the history of biochemistry and of the recent achievements in neuropsychopharmacology.

\section{Evolution of the Biochemistry}

Biochemistry was important to the very beginnings of life on Earth, well before organisms had developed any degree of consciousness. Life depends on keeping biochemical processes within their suitable ranges.

Even single-cell organisms are driven in their movements by chemical stimuli (i.e. chemotaxis) [21]. For instance, a bacterium, whose motion in a uniform environment looks like a random walk, is able to direct its movement toward high concentrations of chemoattractants (usually food) in its environment and away from chemorepellents (usually poisons). It is sensitive to chemical changes in its environment.

Multi-cellular organisms adapted the chemical dependencies of the single-celled organisms to meet their more complex needs of behavior for survivability as new sensors signaled changes in their environment. The ancient behavior of drinking water is to satiate the primordial non-conscious instinct of thirst that is the change in the sodium concentration in the cerebrospinal fluid [22].

The evolutionary development of the Autonomic Nervous System (ANS) in pre-conscious creatures is a demonstration of the important role of biochemistry in behavior for improved survivability [23]. The somatosensory system uses chemical substances as well as nerve fibers to convey signals to interactively produce integrated representations of the body state, its environment, and their relationship at any given moment. These representations have structural, electrical, and chemical as well as temporal dimensions. The systems of the Sympathetic Division of the ANS that controls "fight or flight" response use epinephrine and norepinephrine as their 
primary transmitter to stimulate the adrenal medulla. Similarly, the Parasympathetic Division that controls "rest and repose" response uses neurotransmitters to stimulate production of digestive enzymes. All preganglionic autonomic neurons use acetylcholine as their fast excitatory transmitter and many also contain neuropeptides that mediate slow excitatory post-synaptic potentials.

The non-conscious instincts of primal emotions from the chemical modulations used by the ANS were the origins of our sensitivity to changes in our body chemistry that evolved into primary experiential consciousness. Experiential consciousness (i.e. the awareness of changes in the organism's environment and its body state) evolved because it increased the probability of the organism's survivability by improving its response to change.

The brain manages the life of the organism so as to maintain the internal chemical balances. I propose that a change in the profile of the neurochemistry signals a physiological change with a specific meaning (e.g. hunger or happiness), which relates to the animal's welfare and to its behavior.

The neurochemistry is the essential dynamic feature missing from all of the current explanations of the source of feelings in the brain.

\section{Neurochemistry}

Neurochemistry became a research discipline when it was recognized that transmissions of neural signals between and within the body and the brain are chemical as well as electrical. It is an electrical current that conducts an impulse along the length of a nerve axon, but the sensitivity of a neuron to stimulation for synaptic transmission and reception can be altered by a variety of chemicals called neurotransmitters [24].

There are at least fifty different neurotransmitters that modulate and mediate the interactions among the cells, neurons, axons, and dendrites. Some are inhibitory. Some are excitatory. Some neurotransmitters are released non-synaptically and these chemicals modulate or regulate the coordinated activity of neuronal ensembles over broad regions of the brain and over extended periods of time [25]. Rapid activations or deactivations of widely distributed neuronal activities of the brain are achieved with a variety of chemicals released in many parts of the brain. Such modulation of the activity of widespread target neurons through chemical communication is known to have broad influence on behavioral state [26] [27].

Neurochemicals produce a vast array of neural phenomena across the brain because 1) of their sheer number and variety; 2) each neurochemical may have a different effect on different targets or even on the same target under different conditions; 3) neurotransmitters induce secondary actions and interactive feedback; and 4) the sources of stimulation of various neurotransmitters are widely distributed.

Changes in the amount and distribution of one of those transmitters, or even changes in the relative balance of transmitters at a particular site, can influence cortical activity rapidly and profoundly and are known to give rise to states of depression or elation. Studies in neuropsychopharmacology have shown that neurotransmission/receptor activity and biochemical processes as well as neural circuitry influence feelings and behavior.

\section{Neuropsychopharmacology}

Neuropharmacologists study the interactions of neurotransmitters, peptides, hormones, enzymes, and neuromodulators in the nervous systems. They have discovered that chemicals can induce widespread changes in physiological and in psychological functions by intervening in the operations of these neurotransmitters.

Psychoactive drugs exert their sensory and behavioral effects primarily by acting on neurotransmitters that modify one or more aspects of synaptic transmission. Drugs can affect communication between widely separated cells by acting on hormones, which can travel long distances before reaching target cells anywhere in the body.

Psychoactive drugs have been produced that alter moods, personalities, and emotions; alleviate pain; control stress; quell anxiety; suppress the symptoms of schizophrenia; and stimulate pleasure. Endorphins can kill pain and produce a feeling of well being. Oxytocin facilitates social interactions and induces bonding between mating partners. Estrogen, testosterone, and progesterone profoundly affect mood, especially sexual mood. The neurotransmitter dopamine plays a major role in making an experience feel rewarding. In Parkinson's disease, the production of dopamine is less than normal. The serotonergic system is known to play a fundamental role in emotions, and is believed to be a contributor to feelings of well being and happiness [28] [29].

Neuropsychopharmacologists have shown that a change in a neurotransmitter's chemical structure, or an imbalance at any point in the complex processes, can affect feelings, moods, thoughts, behaviors, and mental states. Mood disorders such as stress, anxiety, and obsession-compulsion are typically treated with drugs that enhance 
the concentration of synaptic serotonin. Global levels of consciousness, sleep and wakefulness, are produced by the daily rhythmical releases of chemicals such as serotonin, adenosine, and the hormone melatonin that affect the ability of different cortical regions to communicate for integration. Anesthesiologists use chemical agents to produce and hold at will different levels of consciousness including its total suspension.

I suggest that the inherent sensitivity of all animals to changes in their body chemistry is the origin of experiential consciousness. I have presented a few examples of how this sensitivity has been demonstrated and exploited by neuropsychopharmacologists to effect changes of feelings in humans through the use of drugs acting on neurotransmitters.

\section{Human Consciousness}

Human consciousness is an elaboration of the consciousness of other animals. Where the non-human animals differ from the human is not in whether they are conscious, but in what they are conscious of. What emerged over the course of evolution of life over a vast period of time and hundreds of thousands of generations were the various qualities and dimensions of conscious experience-the contents of consciousness. There is a continuum of the levels of consciousness among living creatures that ranges across a spectrum of elaborations from that of insects to that of primates. The extent of the elaboration of consciousness that evolved in a species at any moment in time is determined by the size, the complexity, and the variability of the world to which that creature's ancestors adapted to survive. Among those adaptations were the sensors developed to recognize the changes in the environment. Those sensors provide data to the creature's brain where they are processed to produce a response to the environmental change that could affect the creature's survivability.

The highest level of consciousness is knowing that you know. Human consciousness is the adaptation of the highest level of consciousness required for living in a world in which the environment of the society of other people is added to the natural environment. Human consciousness gives us the capability for social learning and the social intelligence to behave in our best interests in a complex societal environment. The evolutionary adaptations of all other animals have been primarily slow genetic changes driven by changes in their natural environment. However, it was the changes in their social environment that became the dominant force during the evolution of Homo sapiens.

Biological evolution alone was too slow to generate behaviors adapted to the rapid changes of the social environment. The plasticity of the neurochemistry enables the brain's adaptability to changing sociocultures. Biochemical balances are the extremely flexible and quickly adaptable attributes of neuronal activities that enabled the rapid behavioral, psychological evolution of humans [30]. The adaptations that enabled Homo sapiens to engage in complex social interactions were elaborations of the neurochemical activities. Consequently, while the physical human evolved over tens of millions of years, the modern human-brain's chemistry adapted relatively quickly (in tens of thousands of years) to co-evolve with the increasing complexities of social living.

Humans acquired the characteristics of human consciousness because they evolved in highly connected groups whose members needed efficient communication to maintain group stability. As their societies grew, humans developed the use of language for dialogue into a "sixth" sensor-a sensor of society. The processing system of its signals that co-evolved with this sensor produced an associated awareness (i.e. human consciousness) needed to manage complex social interactions. As Wilson [31] pointed out, human consciousness enables us "to feel empathy for others, to measure the emotions of friends and enemy alike, to judge the intentions of all of them, and to plan a strategy for personal social interactions". Dialogue was the tool with which members of the group communicated to coordinate group action.

The raison d'être of human consciousness is to enable the individual to cope with the environmental challenges imposed by the societal group of which that individual is a participating member. The sensors, senses, and processes (both conscious and non-conscious) underlying experiential consciousness co-evolved as humans adapted to living in a social group by enhancing, elaborating, and embellishing the capabilities of consciousness inherited from their predecessors. In the co-evolution of socioculture, dialogue, and human consciousness, feeling was an elaboration of the inherited sensitivity to changes in the chemical balance that improved awareness of an environmental change.

\section{How Do We Have Feelings?}

The essential feature of experiential consciousness is that it reflects an integrated representation of the event or 
object. For example, the subjective seeing of a color is different from the measurable objective physical processes entailed in sensing the color. The experience of seeing a color entails a fusion of data from multiple sensors stimulated by the color and its context. The data from the color sensor are integrated with data from other sensors of the same event, with memories of past relevant experiences, and with intentions and goals. The feeling of seeing a color is associated with this integrated representation. The feeling can change with changes in the context even if the color itself remains the same. Your feeling of seeing the piles of white snow that you will need to shovel off of your driveway is not quite the same as your feeling of seeing the snow-white gown on your lovely bride as she comes down the aisle. Memories modulate the signals from the sensors of the external scene by causing the release of certain neurotransmitters, thereby affecting the integrated representation.

The integrated mental representation of an event or object is the fusion of the (real or imagined) signals from one or more of the individual's sensors of his or her environment. These also interactively combine with emotions and memories associated with past experiences of the same or similar stimuli. Consequently, a large number of topologically different neuronal groups distributed across many separate cortical regions of the brain interact simultaneously, synchronously, and continuously.

All of the processing of these data from the various sensors and their distribution to other parts of the creature's brain to produce an integrated representation of its world is done non-consciously.

We are consciously aware of only the result of the non-conscious processes that produce the integrated representation of the event or object, not of any of its components or processes. Moreover, it is only the gist (not the details) of the changes in this non-consciously generated integrated representation that is available to consciousness. This characteristic of human consciousness of being aware of only the gist very likely has its roots in the adaptations to cope with life in a societal group. Human consciousness maintains an awareness of only the gists of (non-conscious) integrated representations that are needed to maintain social interaction and to adapt to irregular changes in environment in real time [32].

In particular, this experiential awareness of only the gist is an adaptation of the use of language needed to maintain effective dialogue. The speed of human speech derives from our having brain mechanisms that have evolved for speech production matched to its comprehension. Our conscious experience of the gist of the conversation initiates the autonomic processes for production and understanding of speech. In rapid speech, we usually do not know precisely what we are going to say until we have heard ourselves say it and we may be only momentarily aware of individual words. It is necessary and sufficient to select and interpret only the "essence" of the (possibly) complex mental representation salient to the moment of the ongoing conversation in order to present it within the limited speed of the serial verbal process. This awareness of the gist elicits the conscious experience of knowing what one is saying (or hearing) without (after some experience) being aware of each word.

Aspects of the non-conscious neuronal processes that produce an integrated representation and its gist of an event or object may well be properly modeled by one or more of the speculative concepts that have been proposed such as Edelman's [4] [5] "reentrant loops" and Baars' [7] global workspace and Beck and Eccles' [10] [11] quantum mechanics and Crick and Koch's [13] synchronization. However, these are all about non-conscious processes. The addition of the consideration of neurochemistry offers the possibility of explaining feelings of the gist of an integrated representation.

Fundamental to the fusion of data from widely distributed regions of the brain and body is its reliance on the use of neurotransmitters across the networks of neuronal activities [24]. The neurotransmitters modulate each signal as it is processed from each of the various sensors. The neurotransmitters also modulate each of those signals as it is combined with the others into an integrated representation. Consequently, the dynamic participation of the neurotransmitters in the processes of this fusion quickly and continuously changes the chemical state of the brain. Moreover, the changes in the neurochemical profile correlate with the changes in the integrated representation because of the roles that the neurotransmitters play in generating those changes.

My contribution to the existing speculations is that the quantity and quality of the conscious experience of an event or object are the changes in the distribution profile of the quantities, types, and locations across the brain of the neurotransmitters used to generate the integrated representation of that event or object and its gist.

Of course, the conscious experience is affected by any changes in the underlying non-conscious neuronal activities that generate the integrated representation. Nevertheless, the feeling itself is the change in the chemical profile that was built in the process of generating the integrated representation.

The results of the work in neurochemistry, psychopharmacology, and neuropsychopharmacology, only some 
of which I have cited, are evidence that feelings are controlled and controllable with chemistry. The knowing that we have feelings arises from our inherited sensitivity to transient changes in the body's chemical and neurological profile triggered (consciously or non-consciously) by the generation of an integrated representation of an (internal or external) event.

The chemical balance in the brain does not cause conscious experience. Conscious experience-feeling-is the change in the chemical balance.

\section{Summary and Conclusion}

My model of conscious awareness is distributed, highly integrated, dynamic systems of populations of cortical and subcortical neuronal physical, electrical, and chemical structures and organs throughout the brain. Nonconscious neuronal activities process and merge signals from sensors into an integrated mental representation of an event or object. Chemical neurotransmitters play an essential role communicating among cells across the brain participating in these processes and they are key to the flexibility of connectivity and overlapping activity.

The how of conscious experience is the change in the profile of the neurochemicals that act as messengers between cells, or of the electrochemical waves in nerves and neurons connecting distant parts together to enable selective and transient holistic representations of events and objects.

We know that a chemical can influence the sensation of, for example, pain. Does it not, therefore, make sense to suggest that the feeling (i.e. experiential consciousness) of pain is neurochemical?

\section{References}

[1] Descartes, R. (1641) Meditations on First Philosophy. In: Cottingham, J., Stoothoff, R. and Murdoch, D., Eds., The Philosophical Writings of René Descartes, Vol. II, Cambridge University Press, Cambridge, 1-62.

[2] René, D. (1647) Les passions de l'âme (The Passions of the Soul). In: Cottingham, J., Stoothoff, R. and Murdoch, D., Eds., The Philosophical Writings of Descartes, Vol. I, Cambridge University Press, Cambridge, 325-383.

[3] Chalmers, D.J. (1995) Facing up to the Problem of Consciousness. Journal of Consciousness Studies, 2, 200-219.

[4] Edelman, G.M. (1987) Neural Darwinism: The Theory of Neuronal Group Selection. Basic Books, Harper Collins Publishers, New York.

[5] Edelman, G.M. (1989) The Remembered Present. Basic Books, Harper Collins Publishers, New York.

[6] Humphrey, N. (1992) A History of the Mind. Simon \& Schuster, New York. http://dx.doi.org/10.1007/978-1-4419-8544-6

[7] Baars, B.J. (1988) A Cognitive Theory of Consciousness. Cambridge University Press, Cambridge.

[8] Penrose, R. (1989) The Emperor's New Mind. Oxford University Press, Oxford.

[9] Penrose, R. (1994) Shadows of the Mind. Oxford University Press, Oxford.

[10] Beck, F. and Eccles, J.C. (1992) Quantum Aspects of Brain Activity and the Role of Consciousness. Proceedings of the National Academy of Sciences of the United States of America, 89, 11357-11361. http://dx.doi.org/10.1073/pnas.89.23.11357

[11] Beck, F. and Eccles, J.C. (2003) Quantum Processes in the Brain: A Scientific Basis of Consciousness. In: Osaka, N., Ed., Neural Basis of Consciousness, John Benjamins, Amsterdam and Philadelphia, 49-141.

[12] Stapp, H. (2007) Quantum Mechanical Theories of Consciousness. In: Velmans, M. and Schneider, S., Eds., The Blackwell Companion to Consciousness, Blackwell, Malden, 300-312. http://dx.doi.org/10.1002/9780470751466.ch24

[13] Crick, F. and Koch, C. (1990) Towards a Neurobiological Theory of Consciousness. Seminars Neurosciences, 2, 263275.

[14] Nagel, T. (1974) What Is It Like to Be a Bat? Philosophical Review, 83, 435-456. http://dx.doi.org/10.2307/2183914

[15] Dennett, D. C. (1991) Consciousness Explained. Little, Brown and Company, Boston.

[16] Chalmers, D.J. (1995b) Absent Qualia, Fading Qualia, Dancing Qualia. In: Metzinger, T., Ed., Conscious Experience, Ferdinand Schöningh Verlag GmbH, Paderborn, 309-328.

[17] Tye, M. (2000) Consciousness, Color and Content. The MIT Press, Cambridge.

[18] Elliot, A.J. and Maier, M.A. (2007) Color and Psychological Functioning. Current Directions in Psychological Science, 16, 250-254. http://dx.doi.org/10.1111/j.1467-8721.2007.00514.x

[19] Van den Tol, A.J.M. and Edwards, J. (2013) Exploring a Rationale for Choosing to Listen to Sad Music When Feeling Sad. Psychology of Music, 41, 440-465. 
http://dx.doi.org/10.1177/0305735611430433

[20] Koch, C. (2009) The Will to Power. Scientific American Mind, 20, 20-21. http://dx.doi.org/10.1038/scientificamericanmind0909-20

[21] Macnab, R.M. and Koshland Jr., D.E. (1972) The Gradient-Sensing Mechanism in Bacterial Chemotaxis. Proceedings of the National Academy of Sciences of the United States of America, 69, 2509-2512. http://dx.doi.org/10.1073/pnas.69.9.2509

[22] Denton, D. (2005) The Primordial Emotions: The Dawning of Consciousness. Oxford University Press, Oxford.

[23] Schmidt, A. and Thews, G. (1989). Autonomic Nervous System. In: Janig, W., Ed., Human Physiology, 2nd Edition, Springer-Verlag, New York, 333-370.

[24] Siegel, G.J., Albers, R.W., Brady, S.T. and Price, D.L. (2006) Basic Neurochemistry. 7th Editon, Elsevier Academic Press, Waltham.

[25] Florey, E. (1960) Physiological Evidence for Naturally Occurring Inhibitory Substances. In: Roberts, E., Baxter, C.F., van Harreveld, A., Wiersma, C.A.G., Adey, W.R. and Killam, K.F., Eds., Inhibitions of the Nervous System and Gamma-Aminobutyric Acid, Pergammon Press, Oxford, 72-84.

[26] Dismukes, R.K. (1979) New Concepts of Molecular Communication among Neurons. Journal of Behavioral and Brain Science, 2, 409-448. http://dx.doi.org/10.1017/S0140525X00063317

[27] Damasio, A. (1994) Descartes’ Error: Emotion, Reason, and the Human Brain. G.P. Putnam’s Sons, New York.

[28] Wrobel, S. (2007) Science, Serotonin, and Sadness: The Biology of Antidepressants: A Series for the Public. The FASEB Journal, 21, 3404-3417. http://dx.doi.org/10.1096/fj.07-1102ufm

[29] Meyer, J.S. and Quenzer, L.F. (2005) Psychopharmacology: Drugs, the Brain, and Behavior. Sinauer Associates, Sunderland.

[30] Alkon, D.L. (1992) Memory’s Voice: Deciphering the Mind-Brain Code. Harper-Collins Publishers, New York.

[31] Wilson, E.O. (2012) The Social Conquest of Earth. Liveright Publishing Corporation, New York.

[32] Kirk, R. (1995) How Is Consciousness Possible? In: Metzinger, T., Ed., Conscious Experience, Imprint Academic, Ferdinand Schöningh Verlag GmbH, Paderborn. 